Military Technical College, Kobry El-Kobbah, Cairo, Egypt

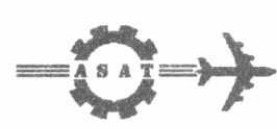

$9^{\text {th }}$ International Conference

On Aerospace Sciences \& Aviation Technology

\title{
PERFORMANCE MODELING OF TAKEOFF/LANDING FOR KT-1 AIRCRAFT
}

M.S. KIM ${ }^{+}$J.H. KIM ${ }^{++}$and J.Y. PARK

\section{ABSTRACT}

A study on the extraction of KT-1 takeoff/landing performance was conducted through the development of the simulation program, which was validated by flight test. Longitudinal 3 DOF equations of motion were solved in conjunction with the modeling of aerodynamics, thrust, landing gear dynamics, control scheme, etc. to simulate the KT-1 trajectories and attitudes during takeoff/landing flight tests. In flight tests, the takeoff/landing trajectories were measured using grid and kinetheodolite tracking method and the grid method was shown to be very useful to measure the trajectories even though very simple. The simulated takeoff/landing distances at test conditions showed little discrepancy as compared to those of flight tests and the simulated control surface displacements, angle of attack, pitch attitude, acceleration also showed good agreements with test data.

\section{KEY WORDS}

Takeoff/landing Performance, KT-1, Simulation, Flight Test, Feedback Control, Simulation Model

\section{NOMENCLATURE}

Cx Aerodynamic Coefficients (e.g., CL, CD, CM)

$\mathrm{K} \quad$ Control Gain

N Landing Gear Reaction Force

$\mathrm{T} \quad$ Thrust

$V_{s e} \quad$ Aft-Stick Velocity at Takeoff

Se Elevator Angle

$\gamma \quad$ Flight Path Angle

$\theta \quad$ Pitch Attitude

$\mu \quad$ Friction Coefficient

+ Agency for Defense Development, Daejeon, Republic of Korea

Korea Aerospace Industries, LTD., Sachon, Republic of Korea 


\section{INTRODUCTION}

The KT-1 aircraft is a turboprop trainer with 950 SHP, which was recently developed in Republic of Korea. The flight performances of the KT-1 have been analyzed, flighttested and successfully demonstrated to the requirements including the takeoff/landing performance. The takeoff/landing is the critical flight regimes in the safety point of view, and these performances were especially focused and flightdemonstrated.

Generally a number of tests are required to provide more credible data to the specific requirements for the operational users. A common method to provide the takeoff/landing data for an AFM is the data reduction and expansion using the typical analytic relations[1,2], which are based on the statistical data of similar class aircrafts. However, some problems were encountered when these procedures were applied to the takeoff/landing data of the KT-1[3]: The correction of thrust, density and weight exhibited an unexpectedly high degree of scatter. It was also realized that a refinement in the methodology was required to account for deviations due to piloting technique.

Hence, to provide more confident takeoff/landing performance data of KT-1 for operational users within the limited number of flight tests, systematic investigations were required. Since an approach based on the modeling and simulation has been widely used to reduce the risk of test and evaluations with a enhanced efficiency $[4,5]$, this approach was chosen to apply to KT-1. In present study, for the extraction of the takeoff/landing performances, a set of simulation program was developed and verified by flight tests as presented in Fig.1. In addition, takeoff and landing trajectories were measured using both grid and kinetheodolite tracking method to substantiate the simulation model.

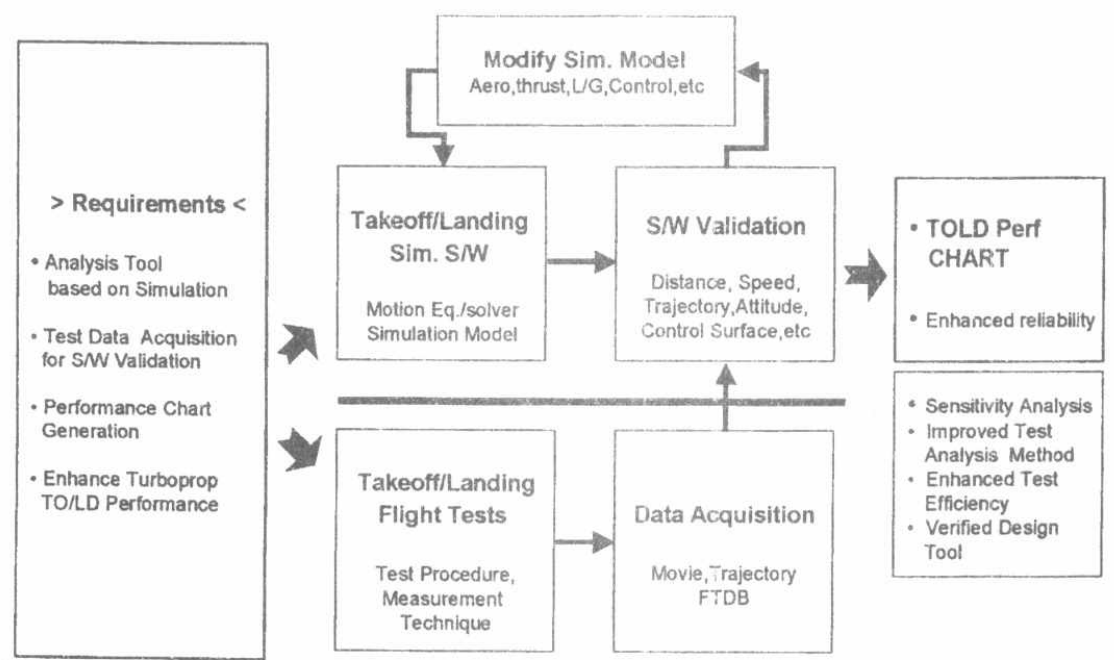

Fig.1. A Procedure for Takeoff/Landing Performance Determination of KT-1 Aircraft 
DESCRIPTION OF KT-1 AIRCRAFT
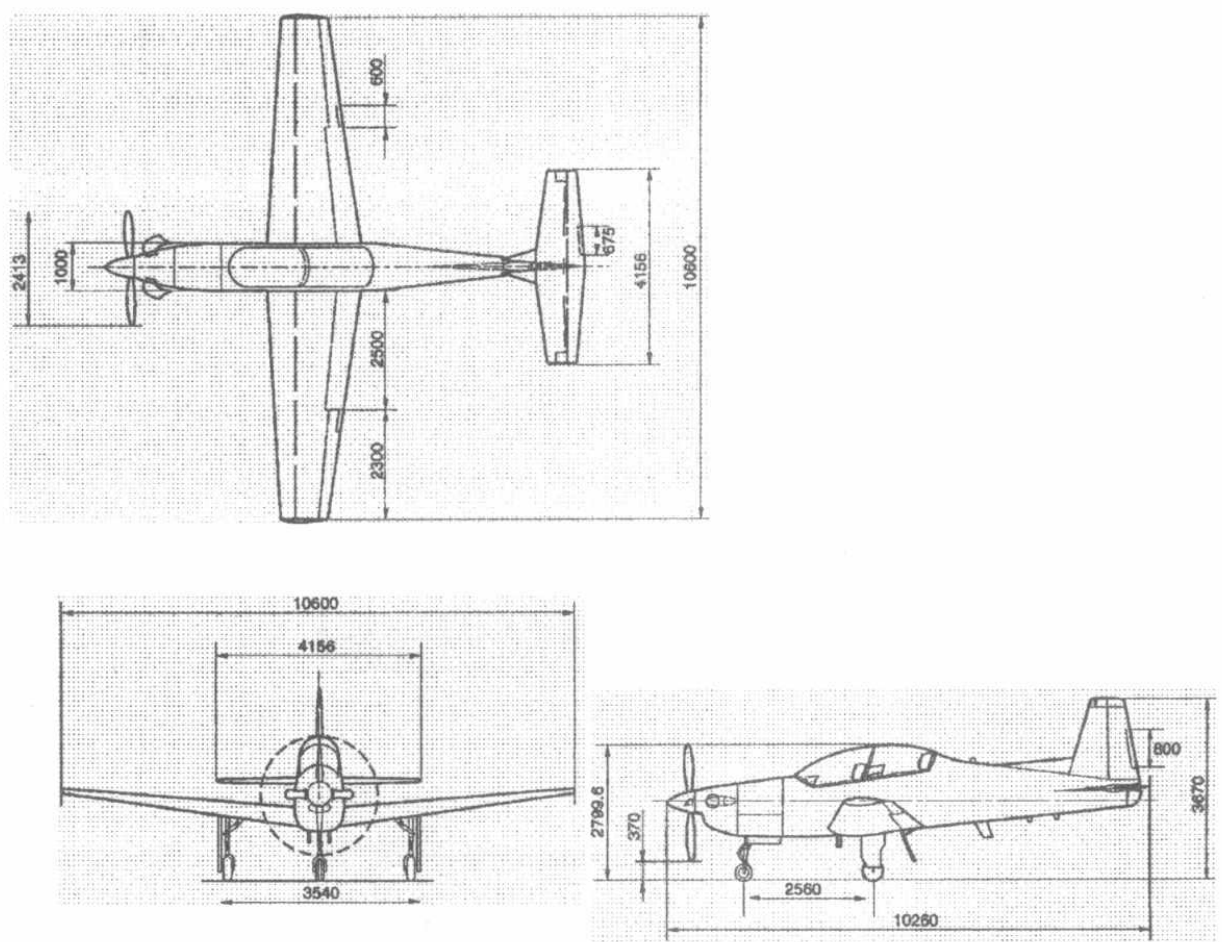

Fig. 2. Three Views of KT-1 Aircraft

$\mathrm{KT}-1$ is a basic trainer fully developed in Korea to replace the venerable Cessna T37s. KT-1 represents a step forward respect to the competitors because is completely new design airplane and is not a derivation from old airplane. The airplane is a low-wing monoplane with a single turboprop engine, P\&WC PT6A62 (950SHP). The structure is semi-monocoque type, with wings and empennage in cantilever construction. The landing gear is retractable tricycle with single wheels. The KT-1 stability and control characteristics are good and in agreement with the FAR 23 and with the MIL-F-8785c requirements. The control forces are harmonized in the complete flight envelope, rolling performance is very high and it is possible to obtain more than $130 \mathrm{deg} / \mathrm{sec}$ rate of roll with low control forces required, longitudinal and directional control forces are positive in all the configuration and at high angle of attack too. Stalling characteristics are safe and the student pilot can recovery from the stall with low loss of altitude. Spinning behavior both erect and inverted are safe and forgiving, even with misapplied control application the airplane does not enter in dangerous conditions, the recovery is obtained in less than 1.5 turns from erect and inverted spin. 


\section{FLIGHT TEST DATA ACQUISITION}

A series of takeoff and landing flight tests were conducted to obtain the trajectories of KT-1 and to substantiate simulation model. Because these tests are strongly influenced by aircraft weight, atmospheric temperature, wind, fieid elevation and piloting technique, a standard piloting technique and takeoff/landing procedures were established before test.

In addition to on-board instrumented parameters, the takeoff/landing trajectories were measured using both grid and kinetheodolite tracking method. A new and efficient concept of photographic tracking on the grid and automatic image processing techniques were applied. As shown in Fig. 3, the azimuth and horizontal angles of the tracking position on the aircraft and four neightooring grid points were digitized and then transformed to trajectory data using the runway information. The results of two different methods agreed well with each other. The current grid method enabled the rotation, liftoff, and touchdown point to be captured visually with a sampling rate of $1 / 30 \mathrm{sec}$, so it could be regarded as cost-effective method without a complex and an expensive device as presented in [6].

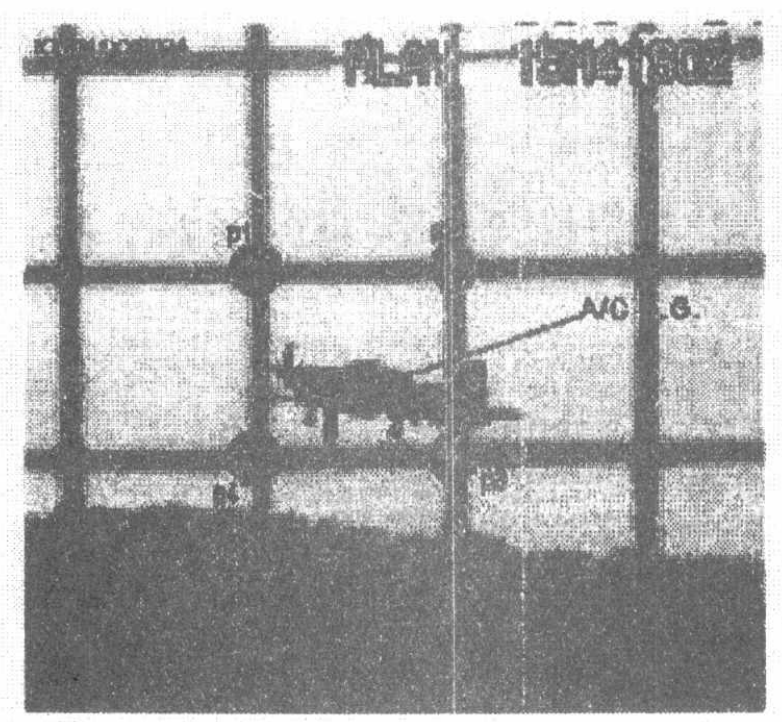

Fig. 3. Trajectory Measurement Using Grid Method 


\section{SIMULATION MODELING}

The equations to describe the motion of the aircraft are three-degree-of freedom, rigid-body equations referenced to the earth fixed coordinate system as follows.

$$
\begin{aligned}
\ddot{x}= & \frac{g}{W}\left[-L \sin \gamma-D \cos \gamma+T_{x} \cos \gamma+T_{z} \sin \gamma-\mu_{1} N_{1}-\mu_{2} N_{2}\right] \\
\ddot{z}= & \frac{g}{W}\left[W-L \cos \gamma+D \sin \gamma-T_{x} \sin \gamma+T_{z} \sin \gamma-N_{1}-N_{2}\right] \\
\ddot{\theta}= & \frac{1}{I_{y y}}\left[M+M_{t}-L e \cos \alpha-D e \sin \alpha-T_{x} h_{t} \cos \alpha+T_{z} h_{t} \sin \alpha\right. \\
& \left.\quad-T_{x} l_{t} \sin \alpha-T_{z} l_{t} \cos \alpha+x_{1} N_{1}-x_{2} N_{2}+z \mu_{1} N_{1}+z \mu_{2} N_{2}\right]
\end{aligned}
$$

where $h_{t}$ and $l_{t}$ denote the moment arms of thrust and $x_{1}, x_{2}$ are the moment arms of landing gear reaction forces, as shown in Fig. 4. The subscript 1 and 2 correspond to nose and main gear respectively. The force and moment terms of above equations are calculated through the various simulation models and the 4-th order Runge-Kutta scheme with a time step of $0.05 \mathrm{sec}$ are used for the time integration of the state derivatives. Initial conditions for takeoff simulation are set through the calculation of static equilibrium. In landing simulation, trim solutions would be found iteratively at specified approach speed and flight path angle.

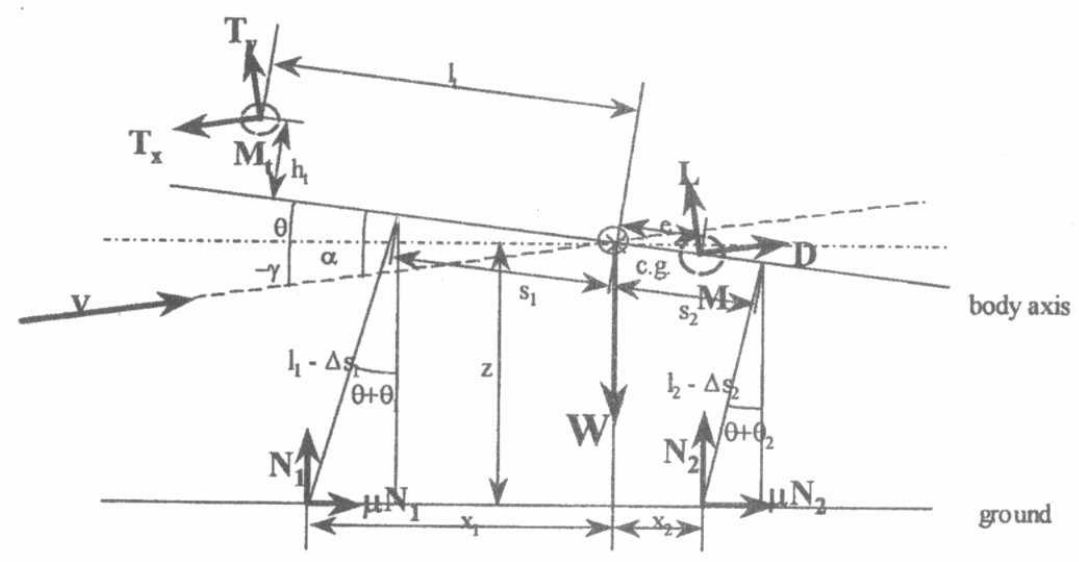

Fig. 4. Free Body Diagram

\section{Aerodynamic Model}

The aerodynamic forces and moments acting on the aircraft were computed by utilizing aerodynamic database, which were based on the wind tunnel data, aerodynamic analyses, and the flight test correlation. The aerodynamic coefficients 
are contained in tabular form as a function of angle of attack, Reynolds number, ground height, thrust coefficient, control surface displacements and etc.

$$
\begin{aligned}
C x=C x_{\text {base }}\left(\alpha, \beta, \delta_{F}\right) & +\Delta C x_{\beta}+\Delta C x_{\delta e}+\Delta C x_{\delta i}+\Delta C x_{\delta r}+\Delta C x_{L G} \\
& +\Delta C x_{S B}+\Delta C x_{G E}+\Delta C x_{X_{i}}+\Delta C x_{R E}
\end{aligned}
$$

Because of the limited wind tunnel test data on ground effect, the height of OGE(Out of Ground Effect) and the effectiveness with respect to the height were modeled using a semi-empirical method[8].

\section{Propulsion Model}

Engine database provided by the manufacturer was reconstructed in the tabular form with respect to speed, altitude, outside atmospheric temperature considering the engine installation loss. Engine database and propeller database were used to calculate the forces and moments aligned to the body axis considering the thrust line orientation.

$$
\begin{aligned}
& T_{X}=\left(T_{P}+T_{j} \theta_{j}\right) \cos \left(\alpha+\alpha_{t}\right) \cos \beta_{t} \\
& T_{z}=-\left(T_{P}+T_{j} \theta_{j}\right) \sin \left(\alpha+\alpha_{t}\right) \cos \beta_{t}
\end{aligned}
$$

In Eq. (3), propeller thrust $\left(T_{p}\right)$ was calculated using the engine and propeller database and jet thrust $\left(T_{j}\right)$ of exhaust gas was added from the engine data.

\section{Landing Gear Model}

The landing gear module computes the reaction forces and moments acting on the aircraft. These forces and moments are based on the pitch attitude, pitch rate, nonlinear spring forces, and damping forces. The stroke displacement( $\Delta S)$ for nonlinear spring forces and stroke displacement rate $(\dot{\Delta} S)$ for nonlinear damping forces were calculated using the landing gear geometric parameter, aircraft pitch and pitch rate. The rolling friction forces and the braking forces were modeled through each friction coefficient.

\section{Control Model}

The elevator control for takeoff simulation was modeled according to the takeoff procedures. During the ground roll until the aft-stick $\operatorname{speed}\left(V_{\delta e}\right)$, the elevator angle was kept as initial setting. The aft-stick speed was acjjusted according to the aircraft weight. After $V_{\delta e}$, the control using the feedback of pitch rate and acceleration was applied to follow the reference pitch-rate command $\left(\dot{\theta}_{r j}\right)$. When the aircraft reached the reference $\operatorname{pitch}\left(\theta_{\text {ref }}\right)$ of about $10^{\circ}$, the control to maintain the pith attitude was applied until the condition of the best angle of climb. 


$$
\begin{array}{lll}
V<V_{\delta e} & : & \delta_{e}=0 \\
V>V_{\delta_{e}} \text { and } \theta<\eta \theta_{\text {ref }} & : & \delta_{e}=\delta_{e}^{o}+K_{11} \ddot{\theta}-K_{12}\left(\dot{\theta}_{\text {ref }}-\dot{\theta}\right) \\
\theta>\eta \theta_{\text {ref }} & : & \delta_{e}=\delta_{e}^{o}+K_{21} \dot{\theta}-K_{22}\left(\theta_{\text {ref }}-\theta\right)
\end{array}
$$

where $\mathrm{K}$ is control gain and $\eta$ is a factor to avoid the numerical overshoot.

To simulate flare and touchdown, landing segments were divided into two by the pitch rate characteristics. A close examination of test results revealed that airborne pahse of landing maneuver could be represented by two constant pitch rates. The elevator angles for these maneuvers were calculated with the feedback of pitch rate as described in Eq. (9) and (10). The reference command values of $0.4 \mathrm{deg} / \mathrm{sec}$ before flare and $1.0 \mathrm{deg} / \mathrm{sec}$ during flare which derived from flight tests could well represent the KT-1 landing characteristics.

$$
\begin{aligned}
& H>H_{F L} \quad: \quad \delta_{e}=\delta_{e}^{o}+K_{11} \ddot{\theta}-K_{12}\left(\dot{\theta}_{\text {ref } 1}-\dot{\theta}\right) \\
& H<H_{r L} \quad: \quad \delta_{e}=\delta_{e}^{o}+K_{11} \ddot{\theta}-K_{12}\left(\dot{\theta}_{\text {ref } 2}-\dot{\theta}\right)
\end{aligned}
$$

After touchdown, aerodynamic braking which was modeled by pitch-hold control could be applied selectively until the wheel brake-on speed.

\section{SIMULATION VALIDATION}

The takeoff/landing simulations were performed at test conditions and compared with flight data. For most of the comparisons, reasonably good agreements were obtained. Fig. 5 . shows that the measured trajectories by grid and theodolite method also agree well with each other, so these could be used as a reference data for validation.

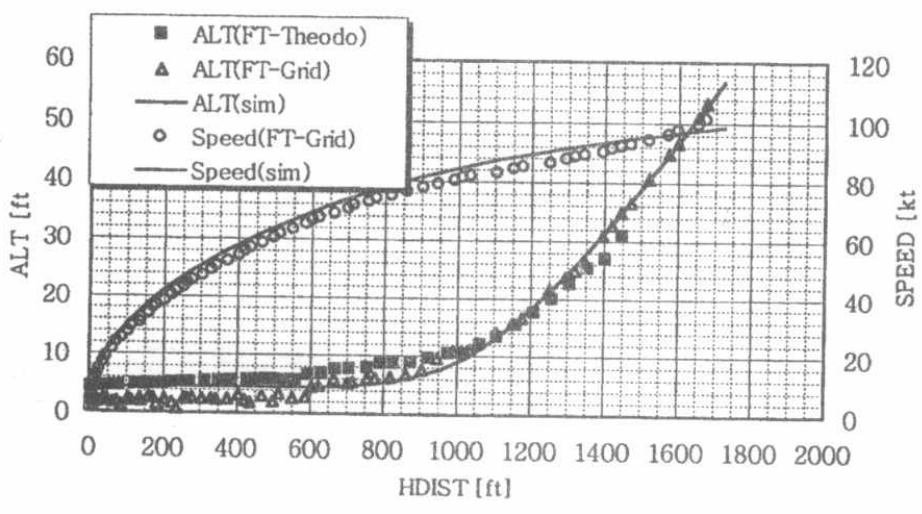

Fig. 5. Takeoff Simulation Validation 
The simulated takeoff ground roll distance at test conditions $(W=5905 \mathrm{lb}$, Head Wind $=4 \mathrm{kt}, T=5^{\circ} \mathrm{C}$ ) showed little discrepancy as compared to flight test. The resultant trajectory, control surface displacements, angle of attack, pitch attitude, acceleration, etc. as shown in Fig. 6. also showed good agreements with flight test data, so the developed simulation model could be regarded as valid.
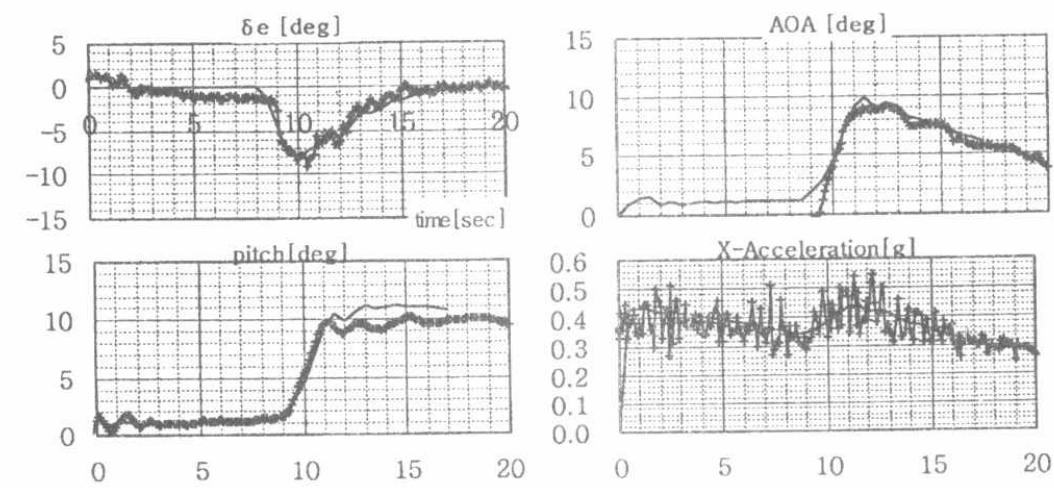

Fig. 6. Takeoff Parameter Validation

A comparison of landing simulation in Figs. 7 and 8 also show that satisfactory representation of landing flight characteristics was obtained. The reference command values of $0.4 \mathrm{deg} / \mathrm{sec}$ before flare and $1.0 \mathrm{deg} / \mathrm{sec}$ of pitch rate during flare enabled the nonlinear flaring trajectory to be well captured.

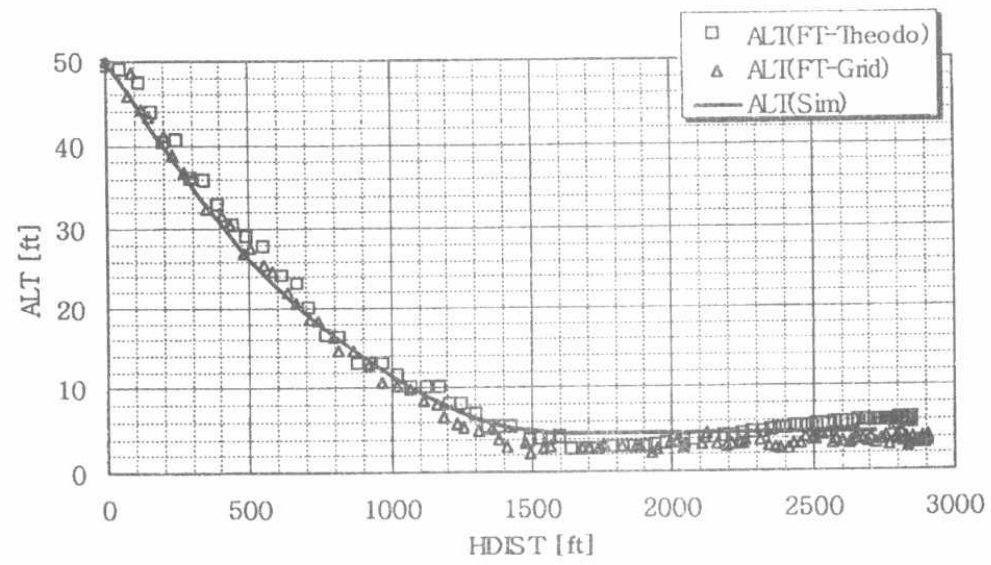

Fig. 7. Landing Simulation Validation 
The resultant airborne distance agree well with flight test and the comparison of the other parameters, such as $a, \theta, \delta \mathrm{e}$, sink rate, etc, also showed good agreements with test data. In landing test of Figs. 7 and 8 , wheel brake was not used to the maximum extent with a safety concern, so the ground segment could not be compared. Simulation with the direct control input of flight test value showed nearly same results with measured values, so it also could be used to evaluate the effects of pilot technique.
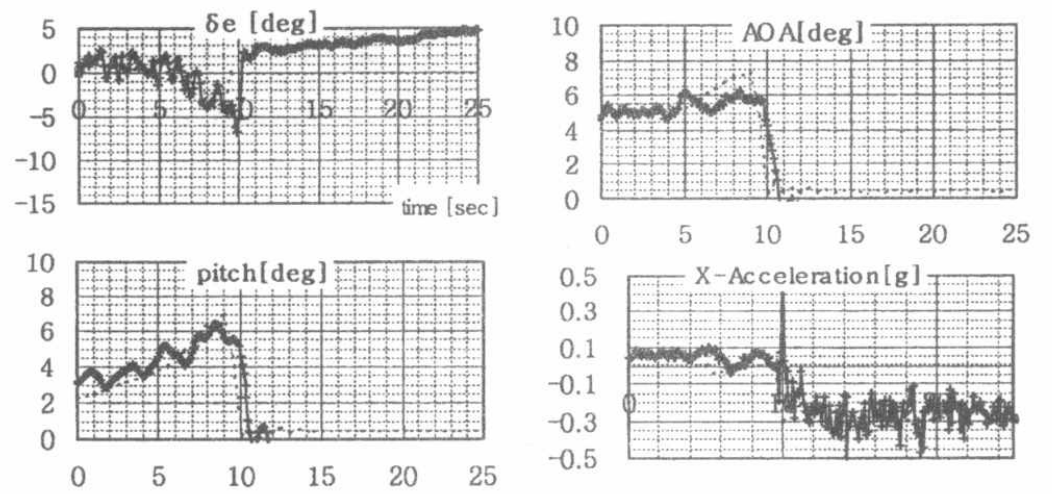

Fig. 8. Landing Parameter Validation

\section{CONCLUSION}

The takeoff and landing performances of the KT-1 were extracted through the development of the simulation program and flight test. The simulation program was verified by flight test data and it showed good agreements on trajectories, attitudes and control surface displacements during takeoff and landing. The control modeling using pitch and pitch rate during takeoff rotation and two constant pitch rate during airborne segment of landing could well represent the takeoff/landing characteristics of the KT-1. Present grid method allowed not only an accurate trajectory measurement but also cost-effectiveness without a complex and an expensive device. The synthesized approach of simulation and flight test demonstration enhanced the insights of takeoff/landing performance of turboprop aircraft. It also enabled to provide the more confident performance data and the standard takeoff/landing procedures for operational users.

\section{REFERENCES}

[1] Dekker, F.E.D. and Lean, D., "Take-Off and Landing Performance," AGARD Flight Test Manual Vol. 1, Chapter-8.

[2] Kohlman, D.L., Flight Test Principles and Practices, Kohlman Systems Research Inc., (1992) 
[3] Park, J.Y. and Kim, M.S., "Take-Off Periormance Test," ASDC-501-990009, (1998).

[4] Riley, D. R., et. Al., "Piloted Simulation Study in an ILS Approach of a TwinPusher Business/Commuter Turboprop Aircraft Configuration," NASA-TM-4516, (1994).

[5] Srivatsan, R., "Development of a Takeoff Performance Monitoring System," NASA-CR-178255, (1987).

[6] P.de Benque d'Agut, et. Al., "Trajectory Measurement for Take-off and Landing Tests and Other Short-Range Applications," AGARD-AG-160, Vol.16, (1985)

[7] Kim, M.S., et. Al., "A Study on the Extraction of KT-1 Takeoff/Landing Performance," MADC-401-001146, ADD Internal report, (2000).

[8] ESDU-72023, "Low-speed Longitudinal Aerodyriamic Characteristics of Aircraft in Ground Effect" 